\title{
APPROACH FOR DIGITALIZATION OF THE TECHNICAL SYSTEMS ON LOGICAL BASIS
}

\author{
Dmitriy Bystrov ${ }^{1}$, Dilfuza Yakubova ${ }^{1}$, Nafisa Xasanova ${ }^{1}$ \\ ${ }^{1}$ Tashkent State Technical University, Department of “Electrical Machines”. 100095, Tashkent, Uzbekistan
}

\begin{abstract}
In the article is given general approach, which can be implemented for digitalization of technical systems as automation in power sector, production sector and etc. Logical inference model is a basis for formalization of various processes. From other hand process of logical inference is a key for such formalization Proposed functioning is a stage question-answer systems proof's, where happens to the list an operation and logic output for realization in computer. So many equations of the logic recorded in digital code.
\end{abstract}

The utterance calculates begin with buildings truth of function to conjunctions $(\wedge)$, disjunctions $(\vee)$, implications $(\rightarrow)$, equivalence $(\sim)$ and negations ( ). The table of importance these function truth ( $\mathrm{t}$ ) and false $(\mathrm{f})$. Here conjunction $(\wedge)$ is not written, for disjunction $(\vee)$ is written by $\operatorname{sign}(+)$ but negation (ך) devil on numeral (for example $\bar{n}, \mathrm{n}=1,2, \ldots$.$) .$

Easy following inter change ability parities are proved $[1,2]$ :

1) $1 \sim 2=12+\overline{1} \overline{2}=(1 \rightarrow 2)(2 \rightarrow 1)$.

2) $1 \rightarrow 2=\overline{1}+2$.

3) $1+2=\overline{1} \rightarrow 2=\overline{\overline{1}} \overline{2}$.

4) $12=\overline{(\overline{1}+\overline{2})}$.

Universal rules of the substitutions are extracted from rules inter change ability parities.

We shall cite an inter change ability parities.

$$
(1 \rightarrow 2 \rightarrow 3)=\overline{1}+(2 \rightarrow 3)=\overline{1}+(\overline{2}+3)=\overline{1}+\overline{2}+3
$$

Change molded gives the way of the adduction truth of function to conjunctive or disjunctive normal forms.

For instance:

$$
\begin{aligned}
& (1 \rightarrow \overline{2}) \sim 3=(\overline{1}+\overline{2}) 3+(\overline{\overline{1}+\overline{2}}) \overline{3}=(\overline{1}+\overline{2}) 3+((12) \overline{3})= \\
& =(\overline{1}+\overline{2}+1)(\overline{1}+\overline{2}+2)(\overline{1}+\overline{2}+\overline{3})(3+1)(3+2)(3+\overline{3}) .
\end{aligned}
$$

So is got conjunctive normal form.

Disjunctive normal form is of the form of

$$
(\overline{1} 3)+(\overline{2} 3)+(12 \overline{3})
$$

Here are taken the laws of distributive.

From normal forms is defined identical true or false molded.

At proof of the theorems, are entered uniquely determined, made and normal forms.

For example:

$$
12 \overline{3}+1 \overline{2} 3+\overline{1} 23+\overline{1} \overline{2} 3 .
$$

We go to interpretation of the deductive logic of the utterance.

It is impossible all identical true expressions to get from small their numbers on base of the rules of the substitution and conclusions.

For instance, let $A=\mathrm{t}, A \rightarrow B=\mathrm{t}$, then $B=\mathrm{t}$. So appears the deductive logic of the utterances.

We write list source molded Gilbert [3].

I. Formulas for implication

1) $1 \rightarrow(2 \rightarrow 1)$.

2) $(1 \rightarrow(1 \rightarrow 2)) \rightarrow(1 \rightarrow 2)$.

3) $(1 \rightarrow 2) \rightarrow((2 \rightarrow 3) \rightarrow(1 \rightarrow 3))$.

II. Formulas for conjunction

1) $12 \rightarrow 1$.

2)12 $\rightarrow 2$.

$3)(1 \rightarrow 2) \rightarrow((1 \rightarrow 3) \rightarrow(1 \rightarrow 23))$.

III. Formulas for disjunction

$$
\begin{aligned}
& 1) 1 \rightarrow(1+2) . \\
& 2) 2 \rightarrow(1+2) . \\
& 3)((1 \rightarrow 3) \rightarrow((2 \rightarrow 3)+((a+b) \rightarrow c)) .
\end{aligned}
$$


IV. Formulas for equivalence

$$
\begin{aligned}
& 1)(1 \sim 2) \rightarrow(1 \rightarrow 2) . \\
& 2)(1 \sim 2) \rightarrow(2 \rightarrow 1) . \\
& 3)(1 \rightarrow 2) \rightarrow((2 \rightarrow 1) \rightarrow(1 \sim 2) .
\end{aligned}
$$

V. Formulas for negation

$$
\begin{aligned}
& \text { 1) }(1 \rightarrow 2) \rightarrow(\overline{2} \rightarrow \overline{1}) . \\
& \text { 2) } 1 \rightarrow \overline{\overline{1}} . \\
& \text { 3) } \overline{\overline{1}} \rightarrow 1 .
\end{aligned}
$$

So is formulated positive logic.

All formulas above written systems are identical true and so of them be removed only true expressions. It is proved that each of molded I-V regardless of all rest molded $\mathrm{I}-\mathrm{V}$ simultaneously is not removed two expressions $A$ and $\rceil A$.

In calculus predicate are entered notions individual variables, is used to rule - a substitutions.

Variable $(A, B)$ take only two importance's "True" and "False ". Besides, all these functions can depend on parameter, belonging to certain area of importance's. They are marked letter of the alphabet [4-6] $(a, b, \ldots)$. The expression of the type $(A(a), A(a, b))$ also take two importance's "True" and "False".

Notion of the substitution is entered as follows: for instance, in formula

$$
A(a, b) \wedge \Theta(a, c) \rightarrow A(c, b)
$$

by substitutions together $A(a, b)$

function $\phi(a, b, c)$ is got

$$
\Phi(a, a, b) \wedge \Theta(a, c) \rightarrow \Phi(a, c, b) .
$$

In calculus predicate is entered quantifier any $\forall x$ and quantifier of existence $\exists x$. Variable these quantifier are called the bounded individual variables.

The substitution in formulas of the calculus predicate happens to by renaming bound variable. Main formulas are added to identical formula of the calculus of the utterances

$$
\begin{aligned}
& \forall x A(x) \rightarrow A(a) \\
& A(a) \rightarrow \exists x A(x)
\end{aligned}
$$

As schemes of the reception new molded is taken

$$
\begin{gathered}
\frac{(A \rightarrow B(a))}{U \rightarrow \forall x B(x)} \\
\frac{B(a) \rightarrow U}{\exists x B(x) \rightarrow U}
\end{gathered}
$$

The study formal output begins the special list of the derived rules with scheduling.

Transition from one formula of the calculus of the utterances to formula of the calculus predicate is executed on the following scheme.

$$
\begin{gathered}
\frac{U \rightarrow(B \rightarrow D(a))}{U \rightarrow(B \rightarrow \forall x D(x))} \\
\frac{U(a)}{\forall x U(x)} \\
\frac{U(a) \rightarrow B(a)}{\forall x U(x) \rightarrow \forall x B(x)} \\
\frac{U(a) \sim B(a)}{\forall x U(x) \sim \forall x B(x)} \\
\exists x U(x) \sim \exists x B(x)
\end{gathered}
$$

At study of the formalism in calculus predicate is entered notion translated and derived rules. These rules shall show on concrete example

1) Rule of the entering quantifier

$$
\frac{U(a, b, c)}{\forall x \exists y \forall z U(x, z, y)} .
$$

2) Rule of the exception quantifier

$$
\frac{\forall y \forall z U(y, z)}{U(a, b)} .
$$

3) Transition from implication and equivalence

$$
\frac{U(b, c) \rightarrow B(b, c)}{\forall x \forall y U(x, y) \rightarrow \forall x \forall y B(x, y)},
$$

$$
\frac{U(a, b, c) \sim B(a, b, c)}{\exists x \exists y \forall x U(x, y, z) \sim \exists x \exists y \forall x B(x, y, z)}
$$

4) Transposition parcel

$$
\frac{U(x) \rightarrow(B(x, y \rightarrow D(x, y))}{\exists x \forall y(B(x, y) \rightarrow(U(x) \rightarrow D(x, y))}
$$

.5) Distribution quantifier

$$
\frac{\forall x y(U(x y) \wedge B(x, y))}{\forall x y U(x, y) \wedge \forall x y B(x, y)} .
$$

6) Introduction quantifier

$$
\frac{\exists x \forall y \exists z(U(x, y) \vee B \vee D(z))}{\exists x \forall y(1(x, y) \vee B \vee \exists z D(z)} \text {.7) }
$$




\section{$\frac{\exists x y z U(x, y, z)}{\forall z y x U(x y z)}$ \\ $\frac{\forall u \forall x \forall y \forall z U(x y z u)}{\forall u \exists x \forall z \forall y U(x y z u)}$.}

8) Change quantifier

$$
\frac{\rceil \forall x \exists y U(x, y)}{\exists x \forall y\rceil U(x, y)} .
$$

In logic of the calculus predicate adduction molded in anticipated form is realized on base molded translated.

$$
\begin{gathered}
\text { We shall take the } \\
\text { example: } \\
\forall x \forall y 1(x, y) \rightarrow \forall x(2(x)+\exists y 3(x, y)) \\
\text { Hereinafter, by rule } 4 \\
\forall y 1(x, y)+\forall x(\overline{2}(x)+\exists y 3(x, y))
\end{gathered}
$$

by rule 6

$$
72(a)+\exists y 3(a, y)
$$

by rule 4

$$
\forall x \exists y(\overline{2}(x)+3(x, y)) .
$$

Conjunction molded

$$
\begin{aligned}
& \qquad x \overline{1}(x, x), \\
& \forall x \forall y \forall z(1(x, y) 1(y z) \rightarrow((x, z)), \\
& \forall x \exists y|(x, y)| \\
& \begin{array}{l}
\text { happens } \quad \text { to } \quad \text { following } \\
\text { type } \\
\forall x y z \exists u(\overline{1}(x, x) \overline{1}(x, y)+\overline{1}(y, z)+1(x, y) 1(x, u)) .
\end{array}
\end{aligned}
$$

\section{Conclusion}

The adduction molded to normal type plays the important role at problems to solubility.

It is proved that any formula of the calculus predicate deductive is such, is anticipated normal form, beside which all quantifiers of existence precede all quantifier ("scale moves cay").

\section{References}

1. Weir, A. Informal proof, formal proof, formalism. The Review of Symbolic Logic, 2016. 9(1), 23-43

2. Jögren, JA note on the relation between formal and informal proof. Acta Analytica, . 2010. 25(4), 447-458.

3. A. Gilibert, P. Bernays. The base mathematicians. The Logical calculuses and formalization of the arithmetics. Moscow "Science", 1979.

4. Hoshimov, F.A., Bakhadirov, I.I., Erejepov, M., Djumamuratov, B. (2019) Development of method for normalizing electricity consumption
E3S
$W e b$
Conf
139

doi:10.1051/e3sconf/201913901074

5. Dmitriy Bystrov, Toirov Olimjon, Giyasov Sanjar, Taniev Mirzokhid, Urokov Sardor, Role of Reengineering in Training of Specialists // NISS2020: Proceedings of the 3rd International Conference on Networking, Information Systems \& Security, March 2020, Morocco, Article No.: 49 P. $1-4$. https://doi.org/10.1145/3386723.3387868

6. Dmitriy Bystrov, Toirov Olimjon, Mustafakulova Gulzoda, Yakubova Dilfuza, Fuzzy Systems for Computational Linguistics and Natural Language // NISS2020: Proceedings of the 3rd International Conference on Networking, Information Systems \& Security, March 2020, Morocco, Article No.: 54, P.1-3. https://doi.org/10.1145/3386723.3387873 